\title{
Chaotic Power System Stabilization Based on Novel Incommensurate Fractional-Order Linear Augmentation Controller
}

\author{
Abdul-Basset A. Al-Hussein ${ }^{\mathbb{D}},{ }^{1}$ Fadhil Rahma Tahir ${ }^{\mathbb{D}},{ }^{1}$ and Karthikeyan Rajagopal $\mathbb{D}^{2}$ \\ ${ }^{1}$ Department of Electrical Engineering, University of Basrah, Basrah, Iraq \\ ${ }^{2}$ Center for Nonlinear Systems, Chennai Institute of Technology, Chennai, India
}

Correspondence should be addressed to Karthikeyan Rajagopal; rkarthiekeyan@gmail.com

Received 30 April 2021; Accepted 11 September 2021; Published 29 September 2021

Academic Editor: Guilherme Ferraz de Arruda

Copyright (c) 2021 Abdul-Basset A. Al-Hussein et al. This is an open access article distributed under the Creative Commons Attribution License, which permits unrestricted use, distribution, and reproduction in any medium, provided the original work is properly cited.

\begin{abstract}
The nonlinear dynamics of an incommensurate fractional-order single-machine infinite-bus (SMIB) power system benchmark model are explored and studied by means of modern nonlinear analysis theories, such as bifurcation, chaos, power spectral density (PSD), and bicoherence methods. The effect of incommensurate order derivatives on power system dynamics is presented. The study reveals that the power system undergoes interesting dynamics such as periodic motion, chaotic oscillations, and multistability whenever the system parameter values fall into particular ranges. A new fractionalorder linear augmentation-based control scheme is applied to damp out the power system's chaotic oscillation, change the stability of the coexisting states, and drive the system from multistability to monostability. The stability of the proposed control system is derived using Lyapunov theory. Simulation results confirmed the effectiveness and robustness of the proposed control scheme in damping power system oscillations and achieving good overall performance. The results in this paper will give a better understanding of the nonlinear dynamic behaviors of the incommensurate fractional-order SMIB power system.
\end{abstract}

\section{Introduction}

Power system is a complex nonlinear dynamical system with many components strongly interconnected, such as generators, buses, transformers, and many other kinds of loads and devices. The continuing interconnections of bulk power systems, brought about by economic and environmental pressures, has led to an increasingly complex nonlinear grid that must operate closer to its stability limits. Therefore, recent power systems have to deal with system stability and reliability control challenges in the current and near future. The power system may be subjected to the disturbances of operation, parameter variation, time delay, noise, and uncertainties involved in a system, which may result in chaotic oscillations that can lead to power system failure, such as voltage collapse, angle divergence, or finally catastrophic blackout like what happened in the US in 1966 [1] and many other countries [2-4]. The power system's dynamical behavior with various parameters is usually associated with complex nonlinear electromechanical oscillations. Moreover, due to the growth of power demand, the modern power grids are forced to operate close to their stability limit and for meeting economic and environmental constraints on installing new transmission lines and new power stations.

Different from power systems' low-frequency oscillations, chaotic oscillations are more critical due to a variety of factors that may trigger this behavior. Due to this fact, it is more difficult to damp out this type of oscillations [1]. In the last few decades, there has been a growing interest in investigating the chaotic oscillation in power systems. One of the first studies on chaos and bifurcation in electrical power networks was presented in [5]. The authors used Melnikov 
theory to analyze a second-order power system with two generators. One of the early works is $[6,7]$ which applied a numerical analysis approach to investigate the chaotic phenomena in a simple power system of two interconnected buses. Indian scholars [8] studied the bifurcations in a three-node power system by approximating the soft wind-up limiter using smooth function to simplify the bifurcation analysis. The scholars in [9] revealed the relationship between various instability modes and chaos phenomena in a three-bus power system and studied the routes to chaos in this model. A single-machine infinitebus power systems model withhard limits in the excitation feedback loop and its effect on the Hopfbifurcation and chaos instability has been presented in [10]. The researchers in [11] proposed a finite-time control method to damp out the chaotic oscillation in a chaotic single-machine infinite bus that shows complex nonlinear dynamics at particular operating conditions. Chaotic power oscillation can occur in electrical networks due to many other reasons such as subsynchronous resonance (SSR) and ferroresonance which are two phenomena that cause power oscillation of rotary systems [12]. During SSR, electrical energy is exchanged between generation unit and transmission systems with frequency below the fundamental synchronous frequency. It happens due to electromechanical interaction of a series compensated transmission line with a generator. It results in oscillation in the shaft and power oscillation [13]. Ferroresonance is a nonlinear resonance, which occurs in presence of a saturable nonlinear inductance and capacitance in a circuit with low resistance. It can emerge due to several configurations like breaker failure during opening or closing operation, line and plant outage, and so on. It causes waveform misshaping, power oscillation, and frequency deviation in the network [14]. A new chaotic power system was introduced in [15], and two different controllers were proposed to remove the chaotic oscillations in the rotor speed and angle.

The theory of fractional calculus dates back to the birth of the theory of differential calculus. But it has begun to be applied in engineering and science applications only in recent years due to its inherent complexity $[16,17]$ and as a result of advances in the fields of chaos and fractals, which revealed subtle relationships with the fractional-order calculus concepts [18]. Many engineering systems, such as electromagnetic waves, dielectric polarization, and quantum evolution of complex systems, show some sort of fractionalorder dynamics [19], and therefore fractional-order control theories have gained researchers' attention.

In [20], a two-dimensional commensurate fractionalorder SMIB model has been analyzed and controlled using a simple Lyapunov-based control method. The proposed controller drives the random chaotic oscillation to perform as a periodic motion. Rajagopal et al. [19] discussed the nonlinear behavior of commensurate fractional-order power system, and the results revealed the existence of chaos oscillation which is suppressed using an adaptive sliding mode controller. Even though there are many studies about power system's chaotic dynamical behavior in the integer-order model, fewer literatures are reporting fractional-order modeling, analysis, and control design for the power system. For these purposes and from the aforementioned discussion, in this paper, the chaos and multistability in an incommensurate fractional-order power system with hard limit will be analyzed and controlled. The applied controller, which is an extension of a recently developed control method based on linear augmentation, has been exploited. This is achieved by coupling the incommensurate fractional-order SMIB model, with a linear feedback system consisting of a basic decaying function [21]. This method offers a significant effect in targeting prescribed steady-state solutions [22], suppressing bistability [23], regulating the dynamics of drive response systems [24], and controlling the dynamics of hidden attractors [25], where the linear augmentation control was used to stabilize fixed points of nonlinear oscillators. The proposed control method has the advantages of simple implementation through electronic circuits, and it is preferred in the situation when the controlled system internal parameters or variables are inaccessible.

This paper is organized as follows. Followed by introduction in Section 1, the mathematical preliminary is given in Section 2. The power system model of both integer-order and incommensurate fractional-order SMIBs is discussed in Section 3. Section 4 introduces the novel fractional-order linear augmentation control strategy to stabilize the SMIB power system. Finally, conclusions are drawn in Section 5.

\section{Preliminaries}

There exist many definitions for fractional-order derivatives. In this work, the Grünwald-Letnikov method will be used for solving the power system dynamics. The Grünwald-Letnikov definition of order $q>0$ (GLD) is given as follows [26]:

$$
{ }_{a}^{G L} D_{t}^{q}=\lim _{h \longrightarrow 0} \frac{1}{h^{q}} \sum_{j=0}^{[t-a / h]}(-1)^{j}\left(\begin{array}{l}
q \\
j
\end{array}\right) f(t-j h),
$$

where $f(t)$ is a continuous function, for $t>0,[t-a / h]$ is the integer part, and

$$
\left(\begin{array}{l}
q \\
j
\end{array}\right)=\frac{q !}{j !(q-j) !}=\frac{\Gamma(q+1)}{q(j+1) \Gamma(q-j+1)},
$$

are the binomial coefficients with $\left(\begin{array}{l}q \\ 0\end{array}\right)=1$. This definition form is very useful for finding numerical solutions of fractional-order differential equations [27]. The numerical solution of fractional-order derivatives is based on (3) derived from the Grünwald-Letnikov (GL) definition (1). The used numerical approximation of $q$ - th derivative at the points $k h(k=1 ; 2 ; \ldots)$ has the following explicit relation $[28,29]$ :

$$
k-\frac{L_{m}}{h D_{t_{k}}^{q} f(t)} \approx h^{-q} \sum(-1)^{j}\left(\begin{array}{l}
q \\
j
\end{array}\right) f\left(t_{k-j}\right),
$$


where $L_{m}$ is the "memory length," $t_{k}=k h, h$ is the time step of calculation, and $(-1)^{j}\left(\begin{array}{c}q \\ j\end{array}\right)$ are binomial coefficients that will be denoted as $c_{j}^{k}(j=0,1, \ldots)$. For their calculation, the following expression can be used [30]:

$$
c_{0}^{q}=1, \quad c_{j}^{q}=\left(1-\frac{1+q}{j}\right) c_{j-1}^{q} .
$$

Then, general numerical solution of the fractional-order differential equation

$$
{ }_{a} D_{t}^{q} y(t)=f(y(t), t),
$$

has the following form:

$$
y\left(t_{k}\right)=f\left(y\left(t_{k}\right), t_{k}\right) h^{q}-\sum_{j=v}^{k} c_{j}^{q} y\left(t_{k-j}\right) .
$$

For the memory term expressed by the sum, a "short memory" principle can be used for various $L_{m}$.

\section{Fractional-Order Power System Model}

A fundamental single synchronous generator with an infinite-bus power system and its exciter is presented in Figure 1. The generator excitation system is represented by a single time constant with high gain AVR and the limiter. The system can be described by the following integer-order model (7):

$$
\left\{\begin{array}{l}
\dot{\delta}=2 \pi f_{0} \omega, \\
\dot{\omega}=-\frac{1}{2 H}\left(D \omega-P_{m}+\frac{E^{\prime} V_{0}}{x+x_{d}^{\prime}} \sin \delta\right), \\
\dot{E}^{\prime}=\frac{1}{T_{d 0}^{\prime}}\left(-\frac{x_{d}+x}{x_{d}^{\prime}+x} E^{\prime}+\frac{x_{d}-x_{d}^{\prime}}{x_{d}^{\prime}+x} V_{0} \cos \delta+E_{f d}\right), \\
\dot{E}_{\mathrm{fdr}}=\frac{1}{T_{A}}\left[-K_{A}\left(V-V_{\mathrm{ref}}\right)-\left(E_{\mathrm{fdr}}-E_{f d 0}\right)\right],
\end{array}\right.
$$

where $E_{\mathrm{fdr}}$ is the field control signal, and the output of the automatic voltage regulator (AVR), $E_{\mathrm{fd}}$, is expressed as follows:

$$
E_{f d}= \begin{cases}E_{\mathrm{fd}_{\text {max }}}, & \text { if } E_{\mathrm{fdr}_{1}}>E_{\mathrm{fdr}_{\text {max }}}, \\ E_{\mathrm{fdr}}, & \text { if } E_{\mathrm{fdr}_{\text {min }}} \leq E_{\mathrm{fdr}} \leq E_{\mathrm{fdr}_{\text {max }}}, \\ E_{\mathrm{fd}_{\text {min }},}, & \text { if } E_{\mathrm{fdr}}<E_{\mathrm{fdr}_{\min }},\end{cases}
$$

and the bus voltage at the generator bus terminal is defined as

$$
V=\frac{1}{x+x_{d}^{\prime}} \sqrt{\left[\left(x_{d}^{\prime}+x E^{\prime} \cos \delta\right)^{2}+\left(x E^{\prime} \sin \delta\right)^{2}\right]},
$$

where $\delta$ denotes the rotor angle, $\omega$ and $f_{0}$ represent angular velocity $(\mathrm{rad} / \mathrm{s})$ and the frequency $(\mathrm{Hz}), D$ denotes damping coefficient (N.m.s/rad), $E^{\prime}$ and $V_{0}$ are the generator's transient excitation and initial terminal voltage $(\mathrm{pu})$, respectively, $P_{m}$ denotes the prime mover mechanical power (pu), $x, x d$, and $x_{d}^{\prime}$ stand for the transmission line and $d$-axis reactance and transient $d$-axis reactance $(p u)$ of synchronous machine, $H$ is the equivalent moment of inertia (pu), and $T_{A}$ and $T_{\mathrm{d} 0}^{\prime}$ represent the time constants (s) of the exciter and the armature winding, respectively. The initial conditions $\left(\delta(0), \omega(0), E^{\prime}(0), E_{\mathrm{fdr}}(0)\right)$ are equal to $(1.046,-0.001,1.33,1.9)$ and system parameters are selected as in Appendix A. The phase portraits for different projections of chaotic integer-order power system (7) are shown in Figure 2.

In this work, the incommensurate fractional-order model of power system is considered, where integer-order derivative is replaced by a fractional order as follows:

$$
\left\{\begin{array}{l}
D^{q_{1}} \delta=2 \pi f_{0} \omega, \\
D^{q_{2}} \omega=-\frac{1}{2 H}\left(D \omega-P_{m}+\frac{E^{\prime} V_{0}}{x+x_{d}^{\prime}} \sin \delta\right) \\
D^{q_{3}} E^{\prime}=\frac{1}{T_{d 0}^{\prime}}\left(-\frac{x_{d}+x}{x_{d}^{\prime}+x} E^{\prime}+\frac{x_{d}-x_{d}^{\prime}}{x_{d}^{\prime}+x} V_{0} \cos \delta\right. \\
\left.E_{\mathrm{fd}}\right), \\
D^{q_{4}} E_{\mathrm{fdr}}=\frac{1}{T_{A}}\left[-K_{A}\left(V-V_{\mathrm{ref}}\right)-\left(E_{\mathrm{fdr}}-E_{\mathrm{fd} 0}\right)\right] .
\end{array}\right.
$$

The numerical solution of fractional-order $4 \mathrm{D}$ power system (10) realized by the Grünwald-Letnikov approximation method with step $h$ is given as follows:

$$
\left\{\begin{array}{l}
\delta\left(t_{k}\right)=2 \pi f_{0} \omega\left(t_{k-1}\right) h^{q_{1}}-\sum_{j=v}^{k} c_{j}^{q_{1}} \delta\left(t_{k-j}\right) \\
\omega\left(t_{k}\right)=-\frac{1}{2 H}\left(D \omega\left(t_{k-1}\right)-P_{m}+\frac{E^{\prime}\left(t_{k-1}\right) V_{0}}{x+x_{d}^{\prime}}\right. \\
\left.\sin \delta\left(t_{k}\right)\right) h^{q_{2}}-\sum_{j=v}^{k} c_{j}^{q_{2}} \omega\left(t_{k-j}\right) \\
E^{\prime}\left(t_{k}\right)=\frac{1}{T_{d 0}^{\prime}}\left(-\frac{x_{d}+x}{x_{d}^{\prime}+x} E^{\prime}\left(t_{k-1}\right)+\frac{x_{d}-x_{d}^{\prime}}{x_{d}^{\prime}+x} V_{0}\right. \\
\left.\cos \delta\left(t_{k}\right)+E_{\mathrm{fd}}\left(t_{k-1}\right)\right) h^{q_{3}}-\sum_{j=v}^{k} c_{j}^{q_{3}} E^{\prime}\left(t_{k-j}\right) \\
E_{\mathrm{fdr}}\left(t_{k}\right)=\frac{1}{T_{A}}\left(-K_{A}\left(V\left(t_{k}\right)-V_{\mathrm{ref}}\right)\right. \\
\left.-\left(E_{\mathrm{fdr}}\left(t_{k-1}\right)-E_{\mathrm{fd} 0}\right)\right) h^{q_{4}}-\sum_{j=v}^{k} c_{j}^{q_{4}} E_{\mathrm{efdr}}\left(t_{k-j}\right),
\end{array}\right.
$$




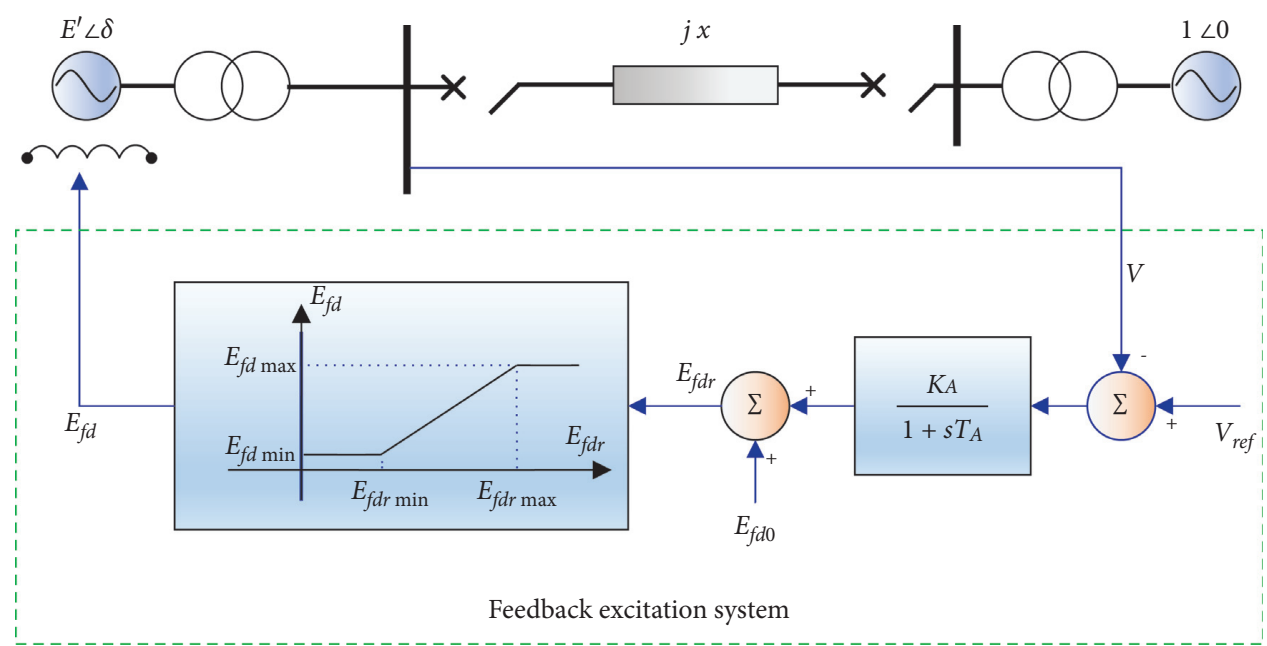

Figure 1: Power system schematic diagram.
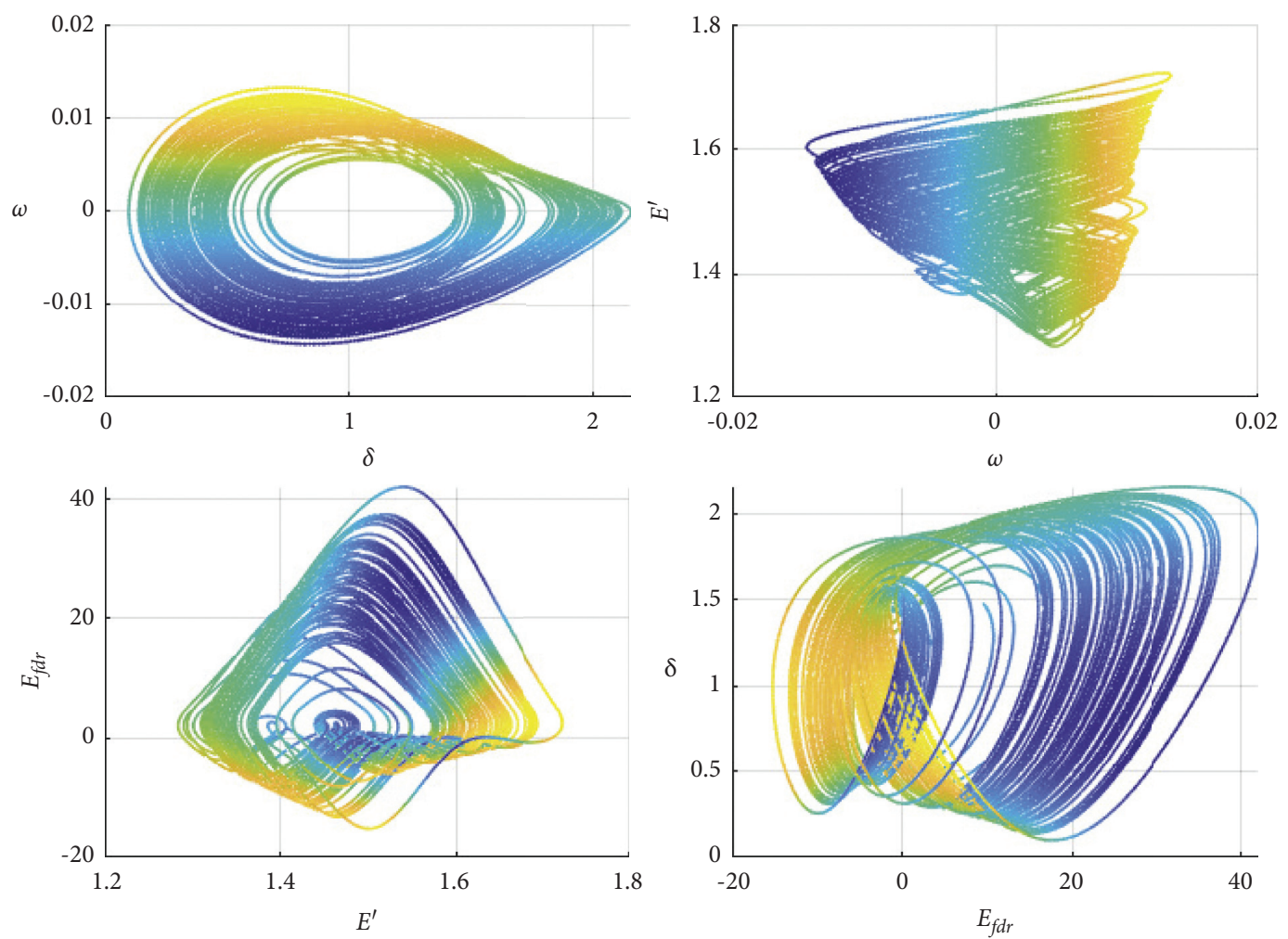

FIGURE 2: Phase portraits of chaotic integer-order four-dimensional power system (7) for different projections.

where $T_{\text {sim }}$ is the total simulation time, $k=1,2,3, \ldots, N$, for $N=[T \operatorname{sim} / h]$ and $\delta(0), \omega(0), E^{\prime}(0), E_{\mathrm{fdr}}(0)$ represent the initial conditions (starting point). The binomial coefficients $\left(c_{j}^{q_{i}}\right)$ are calculated according to relation (4).

3.1. Equilibrium Points and Eigenvalues. The equilibrium points of the fractional-order power system model are the same as those of the integer-order power system. The equilibrium points are found as $(1.0409,0,1.3559,1.9229)$ and $(2.6621,0,2.5362,4.8184)$ [31]. The characteristic equation for the system at the equilibrium can be written as follows:

$$
\operatorname{det}\left(\operatorname{diag}\left[\lambda^{\mathrm{Mq}_{1}}, \lambda^{\mathrm{Mq}_{2}}, \lambda^{\mathrm{Mq}_{3}}, \lambda^{\mathrm{Mq}_{4}}\right]-J_{E}\right)=0,
$$

where $M$ is the lowest common multiple of the denominators $u_{i}$ of $q_{i}^{\prime}$ s where $q_{i}=v_{i} / u_{i}, \operatorname{gcf}\left(u_{i}, v_{i}\right)=1$ and $u_{i}$, and $v_{i} \in Z^{+}$, for $i=1,2, \ldots, n$.

Therefore, for the incommensurate orders $q_{1}=1, q_{2}=1$, $q_{3}=0.999$, and $q_{4}=0.999, M=1000$ and the characteristic equation becomes $\lambda^{3998}+1.1667 \lambda^{2999}+0.15 \lambda^{2998}+$ 
$9.9936 \lambda^{2000}+0.175 \lambda^{1999}+28.707 \lambda^{1998}+1.499 \lambda^{1000}+$ $31.412 \lambda^{999}+473.66$. The condition for the incommensurate fractional-order system to be chaotic is to satisfy the instability measure for the equilibrium points in fractional-order system (IMFOS) as follows [32]:

$$
\frac{\pi}{2 M}-\min _{i}\left(\left|\arg \left(\lambda_{i}\right)\right|\right)>0
$$

which is solved for $0.00016945>0$. The result shows that the incommensurate order of the derivative influences the behavior of the system.. Therefore, the power system (10) tends to be chaotic.

For the same initial condition mentioned before and system parameters given in Appendix A, the incommensurate fractional-order power system phase portraits for different projections are given in Figure 3. The attractors shown in Figure 3 reflect the chaotic nature of the incommensurate fractional-order power system.

The power spectral density and the bicoherence diagram for the fractional-order power system are given in Figure 4. The bicoherence or the normalized bispectrum measures the phase coupling amount in a signal or between two signals. Both bispectrum and bicoherence are used to evaluate the effect of a nonlinear system on the joint probability distribution of the system input. Phase coupling is the estimate of the proportion of energy in every possible pair of frequency components $\left(f_{j}, f_{k}\right)$. Bicoherence results can be used to find coherent signals in extremely noisy data, provided that the coherency remains constant for sufficiently long times, since the noise contribution falls off rapidly with the increasing number of segments $N$.

The chaotic system's auto-bispectrum was given by Pezeshki and his co-workers in [33]. They calculated the auto-bispectrum with the help of Fourier coefficients:

$$
B\left(\omega_{1}, \omega_{2}\right)=E\left[A\left(\omega_{1}\right) A\left(\omega_{2}\right) A^{*}\left(\omega_{1}+\omega_{2}\right)\right],
$$

where $\omega_{1}$ and $\omega_{2}$ are the radian frequencies and $A$ represents the Fourier coefficients of the time series. The normalized magnitude spectrum of the bispectrum known as the squared bicoherence is given by

$$
b\left(\omega_{1}, \omega_{2}\right)=\frac{\left|B\left(\omega_{1}, \omega_{2}\right)\right|^{2}}{P\left(\omega_{1}\right) P\left(\omega_{2}\right) P\left(\omega_{1}+\omega_{2}\right)},
$$

where $P\left(\omega_{1}\right)$ and $P\left(\omega_{2}\right)$ represent the power spectrum at $\omega_{1}$ and $\omega_{2}$.

The bispectrum is calculated by dividing the time series into $M$ segments of length $N_{\text {seg }}$, calculating their biperiodogram and Fourier transforms, and then taking the average over the ensemble. Although the bicoherence is a function of two frequencies, the default output of this function is a one-dimensional output, with the bicoherence refined as a function of only the sum of the two frequencies. From Figure 4, the bicoherence is significantly nonzero and nonconstant, indicating a nonlinear relationship between the states. Moreover, the yellow shades indicate that the nonlinear relationship of the states is not a narrowband process. The power spectral density (PSD) for $100(s)$ time waveforms and sampling period of $0.005(s)$ of the variable $\omega$ is given in Figure 4. The PSD changes uniformly in the whole frequency range, and there is no sharp front. Therefore, the system conforms to chaos characteristics. In Figure 5, the results of the variation with time for two variables $E^{\prime}$ and $E_{\mathrm{fdr}}$ are demonstrated. Analyzing the time waveforms in Figure 5, it can be observed that the system has periodic behavior for $q \leq 0.99$ and tends to exhibit a quasiperiodic or chaotic behavior for $q>0.99$. Therefore, the incommensurate fractional order of the derivative influences significantly the chaotic dynamic behavior of the power system.

To explore the impact of the system parameters on the considered power system and the possibility of occurrence of chaos, the bifurcation plots have been depicted. This diagram reveals the complete dynamical behavior of the system with the variation in the control parameter. The local maxima values of the state variables are plotted with respect to the control parameter By varying the damping coefficient $D$ of the system from 1.48 to 2.0 . Figure 6 shows the bifurcation diagram of the fractional-order power system for $\delta$ state against $D$. As can be observed from Figure 6 , the system takes an inverse period halving exit from the chaotic region. Regions of chaotic oscillations can be seen for $1.48 \leq D \leq 1.58$. That is, for low damping coefficient, the power system can exhibit chaotic oscillation with increasing amplitude when $D$ decreases.

The mechanical power $P_{m}$ has a great influence on stable operation in the power system, which determines the speed of the generator. In order to shed more light on the dynamics of the fractional-order power system in (10), the bifurcation diagrams with respect to the parameter $P_{m} \in[0.95,1.3]$ are shown in Figure 7 for two different initial values. Figure 7 shows that the period-doubling bifurcation is the route to chaos in power system (10) and reveals the existence of two attractors in the range $P_{m} \in$ [0.961.007]. The green line represents a period-1 limit cycle while the magenta line indicates the existence of a period-2 limit cycle. Moreover, the two attractors are shown in the phase portrait plot given in Figure 8.

The coexistence of attractors in nonlinear systems often appears for the same parameter values with different initial conditions, which presents a striking and interesting characteristic of a multistable system. The existence of a switch "jump" motion from period-1 to period- 2 can be observed in Figure 7 due to the effect of the nonsmooth wind-up limiter. The limiter has another interesting and challenging effect on the dynamical behavior of the power system that can be revealed by changing the slope of the limiter, where increasing the gain of the linear part of the limiter strengthens the coexistences of the multistability and induces chaos in fractional-order power system (10). Figure 9 shows the bifurcation diagram of fractional-order power system (10) with limiter linear region gain equal to 10 for two different initial values. It is clear that the slope increase stretches the chaos behavior to a wider range of $P_{m}$. Moreover, Figure 9 illustrates the multistability phenomena where a single-band chaotic attractor and limit cycle attractors coexist for $P_{m} \in[1.060,1.108]$. The phase portrait of system (10) for $P_{m}$ in this range is shown in Figure 10. This confirms that the 

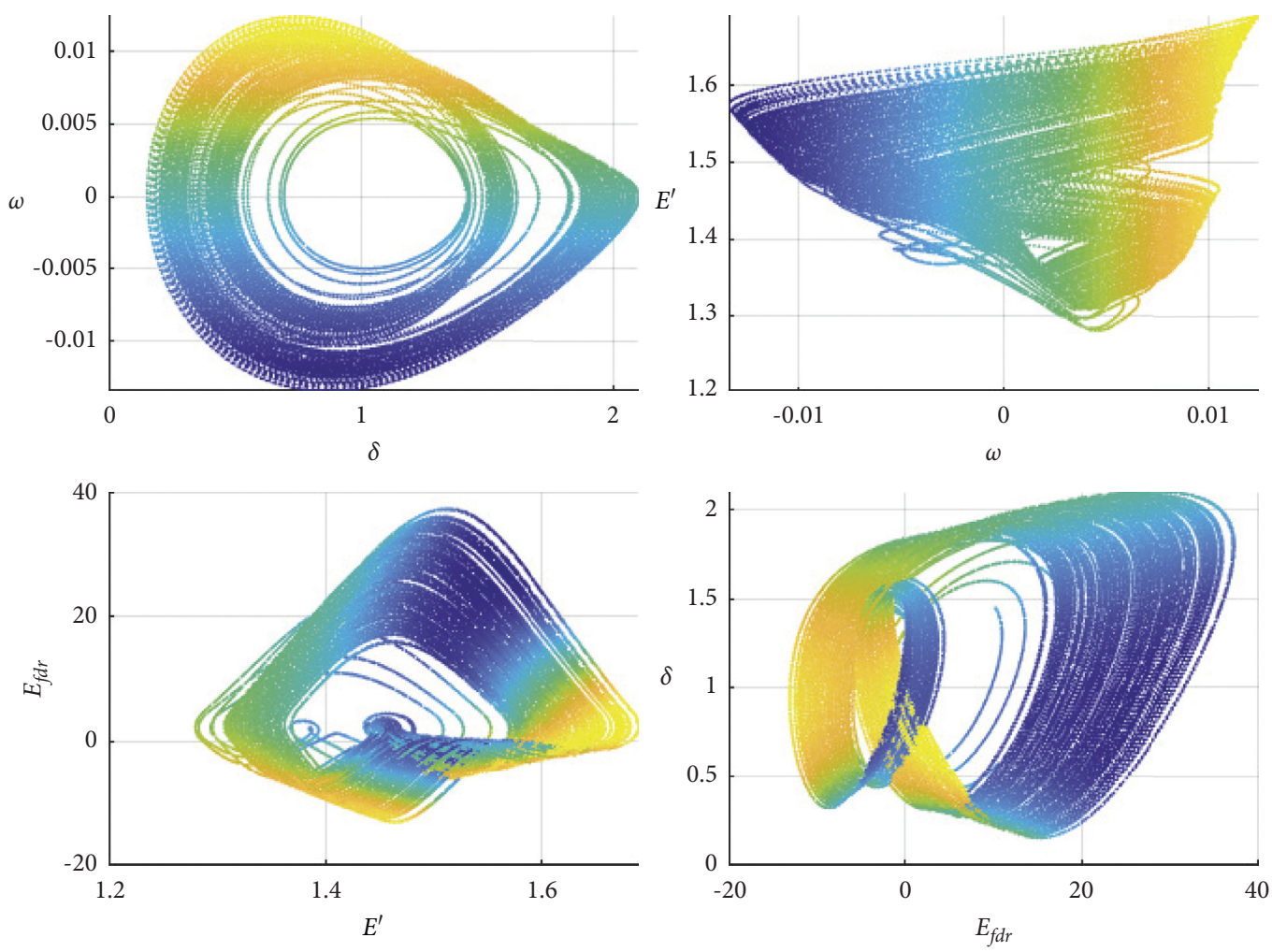

FIGURE 3: Phase portraits of chaotic fractional-order four-dimensional power system (10) for different projections.
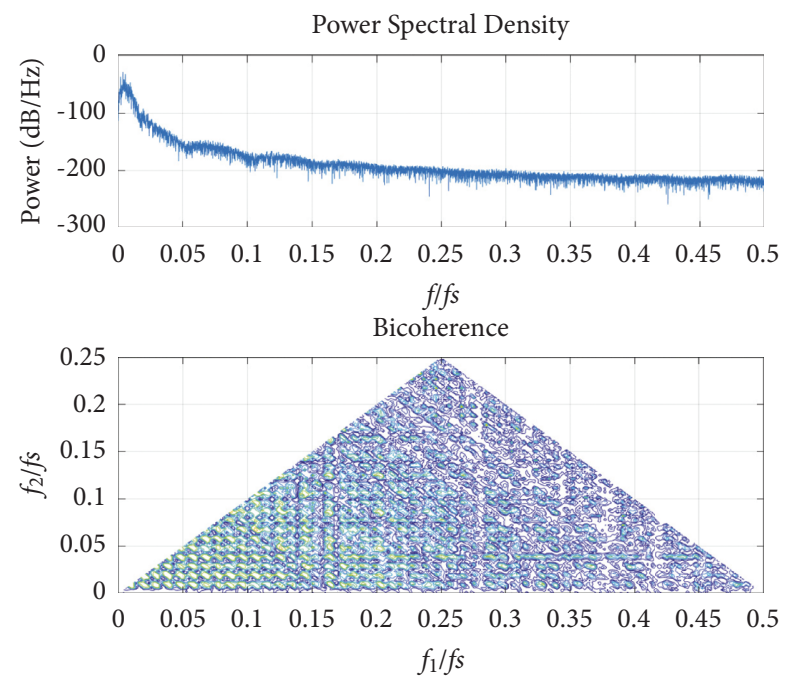

Figure 4: Power spectral density and the bicoherence for fractional-order power system (10).

nonsmooth wind-up limiter has a great effect on the system dynamics not only by inducing chaos but also by changing the nature of the coexisting attractors. From all the aforementioned dynamics and results, it is important to find an appropriate method to damp out the power system chaotic oscillation and control the multistability to enhance the system performance and stabilize the fractional-order power system. In the following section, the fractional-order linear augmentation control method will be presented. To the best of the authors' knowledge, this is the first time to use this method in fractional-order sense.

$$
\left\{\begin{array}{l}
D^{q} X=F(X)+\mu Z \\
D^{q} Z=-\sigma Z-\mu(X-B)
\end{array}\right.
$$

\section{Novel Fractional-Order Linear Augmentation Control Method}

The main goal of the power system control is to damp out power oscillations that occur upon perturbations such as sudden change of loads or in the event of short-circuit 

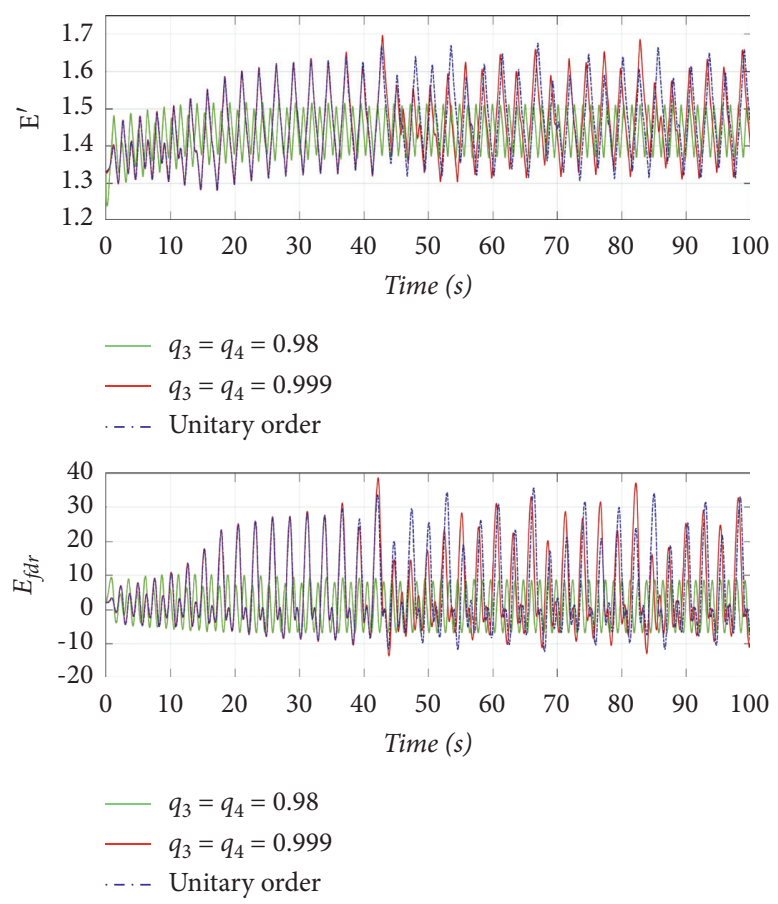

FIgURE 5: Time series responses for fractional-order power system (10) with $q_{1}=q_{2}=1$ and different values for $q_{3}$ and $q_{4}$ as shown in the figure legends.

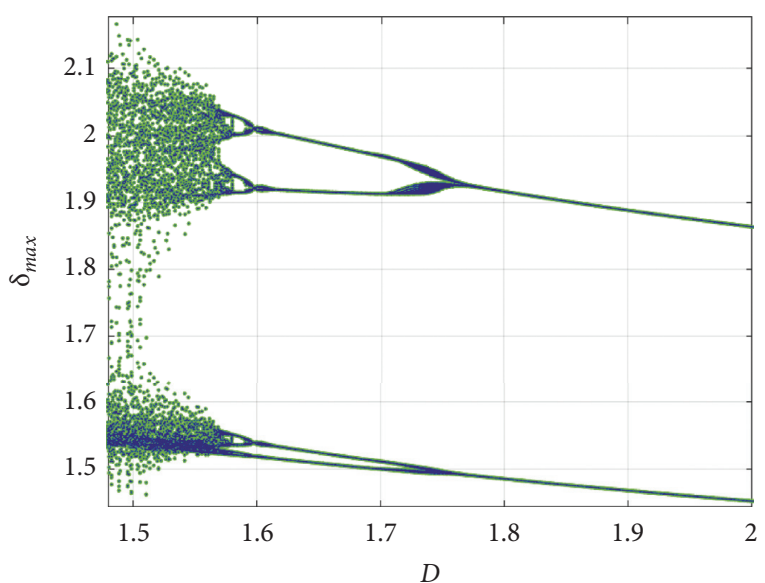

FIGURE 6: Bifurcation diagram for fractional-order power system (10), showing the local maxima for the state $\delta$ with respect to the parameter $D$.

occurrence. These oscillations hamper power flow drastically and may cause an inability to meet power demand or even loss of synchronism that may eventually lead, in the worst case, to blackouts.

Based on the theory of linear augmentation control method [34, 35], this section presents the generalization and extension of this method to handle the fractional-order nonlinear system. The new proposed fractional-order linear augmentation control method is based on coupling the fractional-order nonlinear dynamical system to another fractional-order linear system $(Z)$. Then, by varying the coupling strength between the two fractional-order systems, the control objective can be achieved. The controlled system

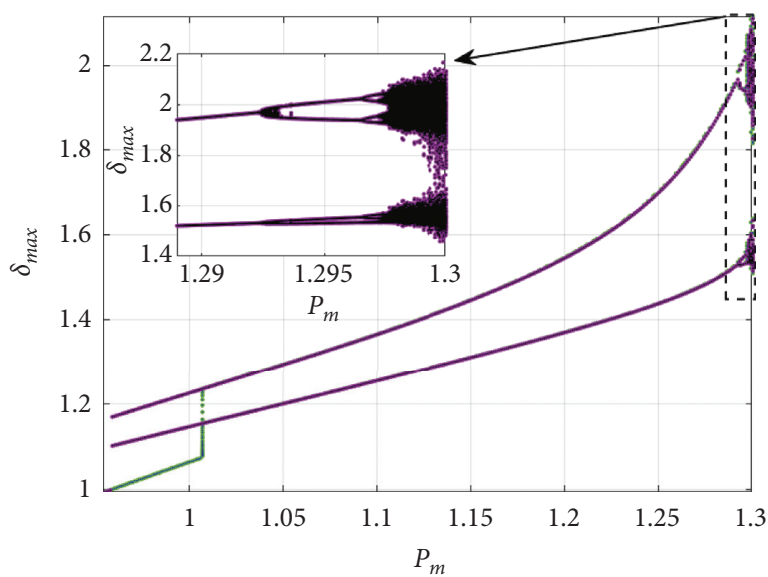

FIGURE 7: Bifurcation diagram for fractional-order power system (10).

can be defined as follows.where $D^{q} X=F(X)$ is a general incommensurate fractional-order nonlinear dynamical system, $X$ is an $\mathrm{m}$-dimensional vector of dynamical variables, and $F(X)$ is its vector field. The parameter $\mu$ represents the coupling strength between the fractional-order nonlinear oscillator and the fractional-order linear control system. The vector $Z$ describes the dynamics of the fractional-order linear system $D^{q} Z=-\sigma Z$, where $\sigma$ exemplifies the decay parameter. In case of no coupling $\mu=0$, the fractional-order linear system approaches exponentially to zero with decaying rate equal to $\sigma$. To prove the previous statement, Lemma 1 [36] will be employed along with Theorem 1 to prove the asymptotic stability of the fractionalorder linear augmentation system. 


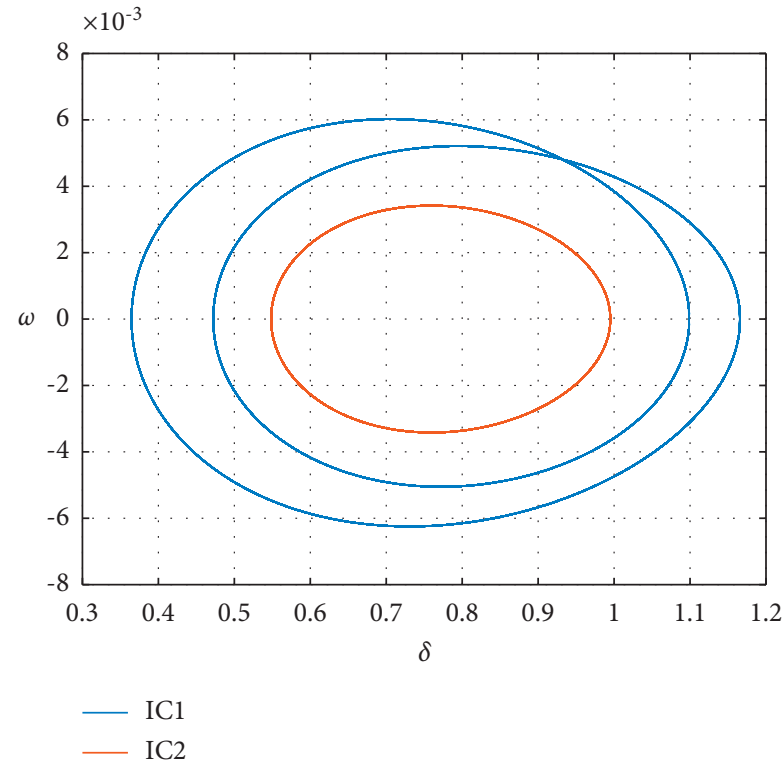

Figure 8: Phase portrait for fractional-order power system (10) at $P_{m}=0.956$ under two different initial conditions.

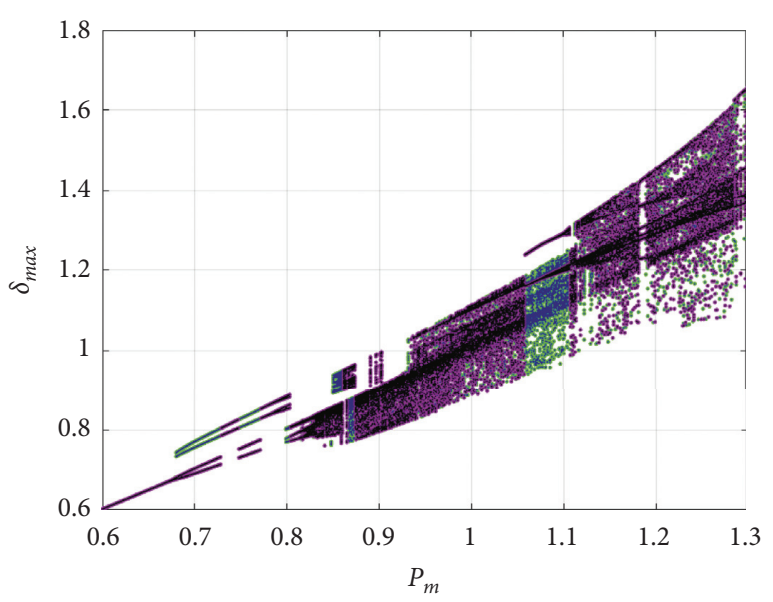

FIgURE 9: Bifurcation diagram for fractional-order power system (10) with limiter slope equal to 10 and two different initial conditions.

Lemma 1. Let $z(t) \in R$ be a continuous and derivable function. Then, for any time instant $t \geq 0$,

$$
\frac{1}{2}^{t_{0}} D_{t}^{q} z^{2}(t) \leqslant z(t)_{t_{0}}^{C} D_{t}^{q} z(t), \quad \forall q \in(0,1) .
$$

Proof. Proving that expression (17) is true is equivalent to proving that

$$
z(t)_{t_{0}}^{C} D_{t}^{q} z(t)-\frac{1}{2}{ }_{t_{0}}^{C} D_{t}^{q} z^{2}(t) \geqslant 0, \quad \forall q \in(0,1),
$$

and it can be written as

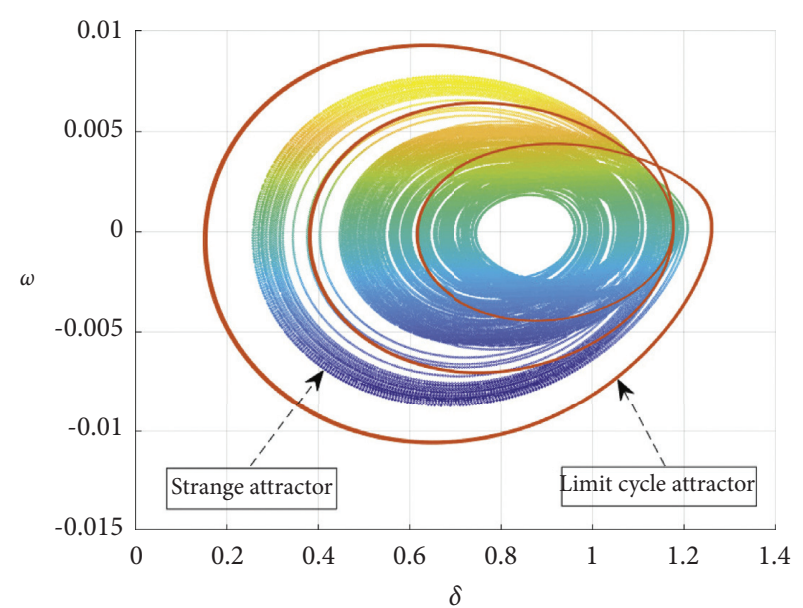

Figure 10: Phase portrait for fractional-order power system (10) at $P_{m}=1.101$, showing coexisting attractors for two different initial conditions.

$$
{ }_{t_{0}}^{C} D_{t}^{q} z(t)=\frac{1}{\Gamma(1-q)} \int_{t_{0}}^{t} \frac{\dot{z}(\tau)}{(t-\tau)^{q}} \mathrm{~d} \tau,
$$

and in the same way,

$$
\frac{1}{2}{ }^{t_{0}} D_{t}^{q} z^{2}(t)=\frac{1}{\Gamma(1-q)} \int_{t_{0}}^{t} \frac{z(\tau) \dot{z}(\tau)}{(t-\tau)^{q}} \mathrm{~d} \tau .
$$

So, expression (18) can be written as

$$
\frac{1}{\Gamma(1-q)} \int_{t_{0}}^{t} \frac{[z(t)-z(\tau)] \dot{z}(\tau)}{(t-\tau)^{q}} \mathrm{~d} \tau \geqslant 0
$$

Define the auxiliary variable as $y(\tau)=z(t)-z(\tau)$, which implies that $y^{\prime}(\tau)=\mathrm{d} y(\tau) / \mathrm{d} \tau=-\mathrm{d} z(\tau) / \mathrm{d} \tau$. In this way, expression (21) can be written as

$$
\frac{1}{\Gamma(1-q)} \int_{t_{0}}^{t} \frac{y(\tau) y^{\prime}(\tau)}{(t-\tau)^{q}} \mathrm{~d} \tau \leqslant 0 .
$$

Let us integrate by parts expression (22) defining

$$
\begin{aligned}
\mathrm{d} u & =y(\tau) y^{\prime}(\tau) \mathrm{d} \tau, \quad u=\frac{1}{2} y^{2}, \\
v & =\frac{1}{\Gamma(1-q)}(t-\tau)^{-q}, \quad \mathrm{~d} v=\frac{q}{\Gamma(1-q)}(t-\tau)^{-q-1} .
\end{aligned}
$$

In that way, expression (22) can be written as

$$
\begin{aligned}
& -\left.\left[\frac{y^{2}(\tau)}{2 \Gamma(1-q)(t-\tau)^{q}}\right]\right|_{\tau=t}+\left[\frac{y_{0}^{2}}{2 \Gamma(1-q)\left(t-t_{0}\right)^{q}}\right] \\
& +\frac{q}{2 \Gamma(1-q)} \int_{t_{0}}^{t} \frac{y^{2}(\tau)}{(t-\tau)^{q+1}} \mathrm{~d} \tau \geqslant 0 .
\end{aligned}
$$

Let us check the first term of expression (24), which has an indetermination at $\tau=t$, so let us analyze the corresponding limit. 


$$
\begin{aligned}
\lim _{\tau \longrightarrow t} & \frac{y^{2}(\tau)}{2 \Gamma(1-q)(t-\tau)^{q}} \\
\quad= & \frac{1}{2 \Gamma(1-q)} \lim _{\tau \longrightarrow t} \frac{[z(t)-z(\tau)]^{2}}{(t-\tau)^{q}} \\
\quad= & \frac{1}{2 \Gamma(1-q)} \lim _{\tau \rightarrow t} \frac{\left[z^{2}(t)-2 z(t) z(\tau)+z^{2}(\tau)\right]}{(t-\tau)^{q}} .
\end{aligned}
$$

Given that the function is derivable, L'Hospital's rule can be applied. Then,

$$
\begin{aligned}
& \frac{1}{2 \Gamma(1-q)} \lim _{\tau \longrightarrow t} \frac{\left[z^{2}(t)-2 z(t) z(\tau)+z^{2}(\tau)\right]}{(t-\tau)^{q}} \\
& \quad=\frac{1}{2 \Gamma(1-q)} \lim _{\tau \longrightarrow t} \frac{[-2 z(t) \dot{z}(\tau)+2 z(\tau) \dot{z}(\tau)]}{-q(t-\tau)^{q^{-1}}} \\
& \quad=\frac{1}{2 \Gamma(1-q)} \lim _{\tau \longrightarrow t} \frac{[2 z(t) \dot{z}(\tau)-2 z(\tau) \dot{z}(\tau)](t-\tau)^{1-q}}{q} \\
& \quad=0 .
\end{aligned}
$$

So, expression (24) is reduced to

$$
\frac{y_{0}^{2}}{2 \Gamma(1-q)\left(t-t_{0}\right)^{q}}+\frac{q}{2 \Gamma(1-q)} \int_{t_{0}}^{t} \frac{y^{2}(\tau)}{(t-\tau)^{q+1}} \mathrm{~d} \tau \geqslant 0 .
$$

Expression (27) is clearly true, and this concludes the proof.

Theorem 1. Consider the following fractional-order system, with $0<q<1$; then, the system is asymptotically stable.

$$
{ }_{t_{0}}^{C} D_{t}^{q} z(t)=-z(t)
$$

Proof. Let us consider the following Lyapunov candidate:

$$
V(z(t))=\frac{1}{2} z^{2}(t)
$$

Now, applying Lemma 1, it can be found that

$$
\begin{aligned}
{ }_{0}^{C} D_{t}^{q} V(z(t)) & =\frac{1}{2}{ }_{0}^{C} D_{t}^{q} z^{2}(t) \\
& \leq z(t){ }_{0}^{C} D_{t}^{q} z(t) \\
& \leq z(t){ }_{0}^{C} D_{t}^{q} z(t) \\
& =-z^{2}(t) .
\end{aligned}
$$

As can be seen from (30), the fractional derivative of the Lyapunov function is negative definite, so it can be concluded that the origin of the system (28) is asymptotically stable.

Now, turn back to the choice of the parameter $B$, which has a very significant role in affecting the desired attractor from the multistable system. $B$ value can be selected as a constant in the vicinity of the prescribed state. Usually, invariant characteristics of the system such as equilibrium points around which the desired attractor is located are preferred. In the special case of hidden attractors in which the attractors are not located in the neighborhood of fixed points, $B$ can be considered as the average of the system's variables (i.e., $B=\sum_{t} X_{t}$ ) [23]. The control strategy described is now applied to the fractional-order power system. The coupling is introduced along the $\omega$ variable with the coupling strength $\mu$, and $Z=[0, z, 0,0]^{T}$ and $B=[0, \beta, 0,0]^{T}$, where $T$ indicates the transpose. The full controlled system dynamics are described in the following equation:

$$
\left\{\begin{array}{l}
D^{q_{1}} \delta=2 \pi f_{0} \omega \\
D^{q_{2}} \omega=-\frac{1}{2 H}\left(D \omega-P_{m}+\frac{E^{\prime} V_{0}}{x+x_{d}^{\prime}} \sin \delta\right)+\mu z \\
D^{q_{2}} z=-\sigma z-\mu(\omega-\beta) \\
D^{q_{3}} E^{\prime}=\frac{1}{T_{d 0}^{\prime}}\left(-\frac{x_{d}+x}{x_{d}^{\prime}+x} E^{\prime}+\frac{x_{d}-x_{d}^{\prime}}{x_{d}^{\prime}+x} V_{0} \cos \delta\right. \\
\left.+E_{\mathrm{fd}}\right) \\
D^{q_{4}} E_{\mathrm{fdr}}=\frac{1}{T_{A}}\left[-K_{A}\left(V-V_{\mathrm{ref}}\right)-\left(E_{\mathrm{fdr}}-E_{\mathrm{fd} 0}\right)\right] .
\end{array}\right.
$$

Owing to this approach, to synthesize the chaos suppression, it is not necessary to control all the states of the model, but it becomes possible to quench the chaos using single scalar coupling. This has the advantage of optimization for resources, when compared with other works in this field, for example, but not limited to [19, 37, 38], where there is a control term for each state in the model to achieve the chaos suppression in the power system. Except for the linear augmentation method, in almost all other existing chaos control methods, the control is applied to one parameter of the system or to the system variable. But generally, it may not be possible to modify the system parameters to remove one of the attractors for all initial conditions. Hence, external control such as the linear augmentation method would be preferred [39].

The results of applying the fractional-order control scheme on the power system are depicted by the bifurcation diagrams in Figure 11 when varying the coupling strength $\mu$ in the range $[0,5]$. Figure 11 shows the transition from chaotic oscillation to monostability behavior at a higher value of the coupling strength parameter. Moreover, it can be observed from Figure 11 that the controlled fractionalorder linear augmentation power system (31) takes an inverse period halving exit from the chaotic region, and the angle amplitude reduced significantly for higher value of the coupling strength parameter $\mu$.

To further investigate the proposed control method, the results of implementing the controller at different cases are presented in Figures 12 and 13. Figure 12 shows the 


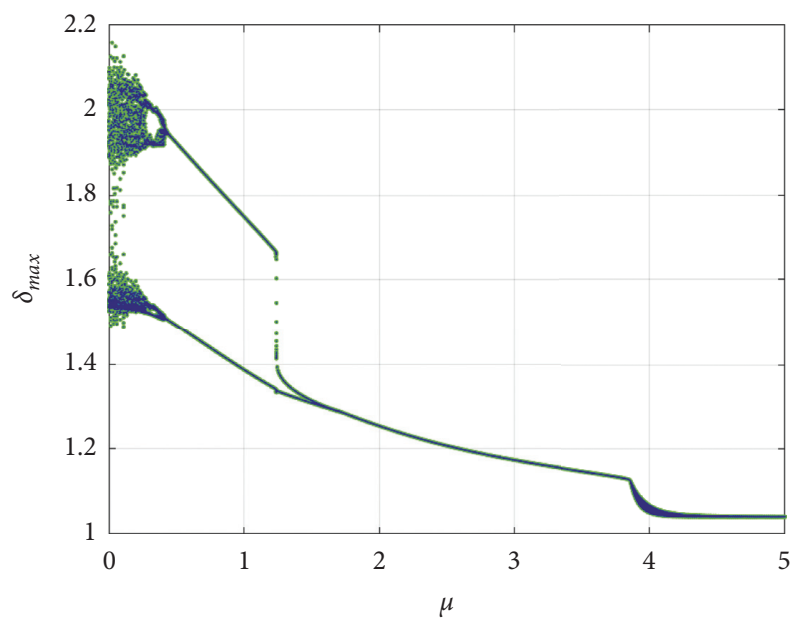

FiguRe 11: Bifurcation diagram for the controlled fractional-order power system (31) by varying the coupling strength parameter $\mu$.
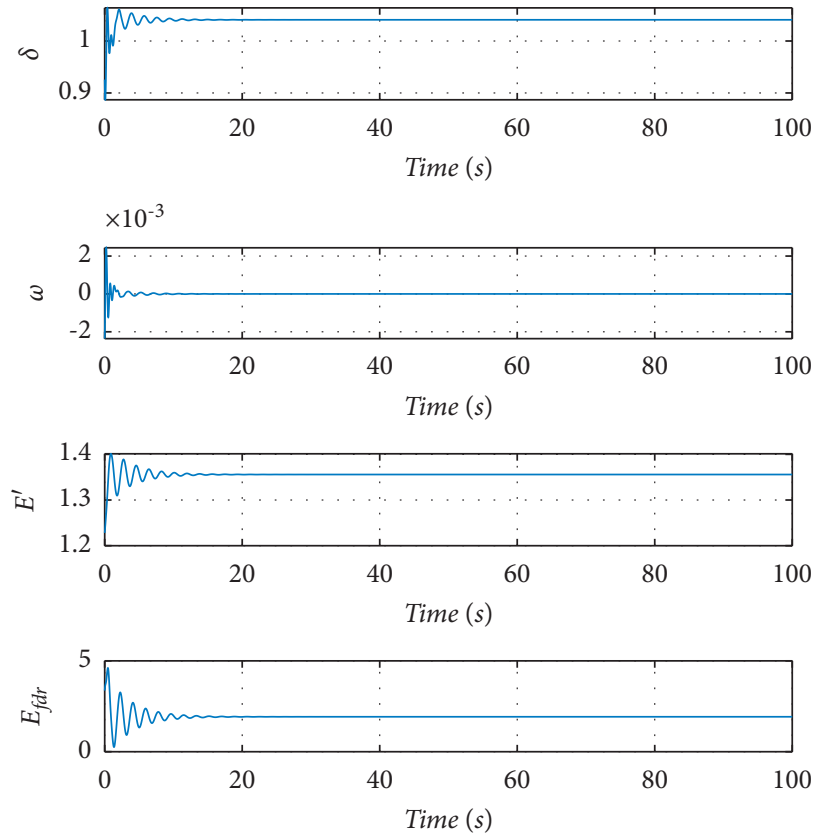

FIgURE 12: Time series responses for controlled fractional-order power system (31) with control in action at $t=0(s)$.

time-domain waveform of state variables of the power system under the proposed controller when it has been applied at the beginning of the simulation at $t=0(s)$. On the other hand, the view of the time-domain waveform of state variables of the power system is shown in Figure 13, when the controller is put into effect at an arbitrary time while the system is in chaotic oscillation state. The two scenarios illustrated the ability of the proposed fractional-order linear augmentation control strategy to reduce the amplitude of the chaotic oscillation, drive system states to their prescribed 

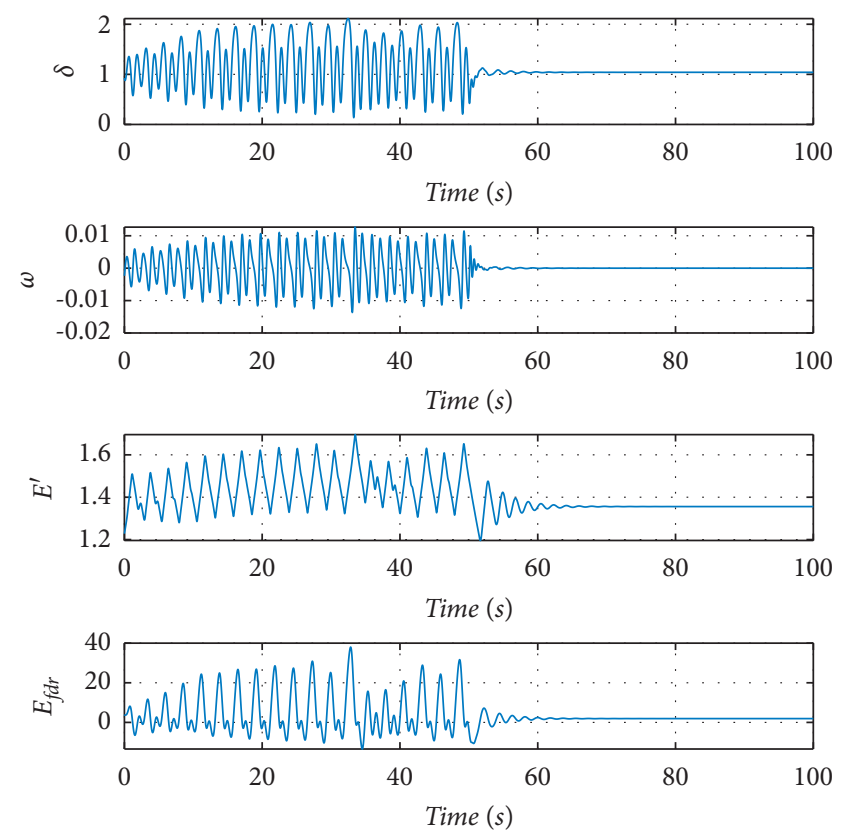

FIGURE 13: Time series responses for controlled fractional-order power system (31) with control in action at $t=50(s)$.

steady-state operating point, and stabilize the whole controlled dynamics of the fractional-order power system. In other words, the proposed controller has good robustness throughout the entire process and achieves the desired control objectives.

\section{Conclusion}

The rich dynamics of a fractional-order power system are investigated in the current paper by varying different system parameters. Based on nonlinear dynamic analysis methods, the rich chaotic behaviors of the power system are investigated and depicted through equilibrium points, power spectral density, bicoherence, bifurcation diagrams, and phase portraits. The derived numerical analysis results are very helpful and worthy in understanding the system behavior. The multistability phenomenon and coexisting periodic/chaotic attractors in the nonlinear fractional-order power system dynamics are revealed for the same system parameters. This represents an interesting and a challenging phenomenon for stability notion and should be seriously considered in control design methodology. A new fractional-order linear augmentation controller is constructed to stabilize unstable states, suppress chaotic oscillation behavior, and achieve monostability for the power system's closed-loop dynamics. The Lyapunov method has been exploited to prove the stability of the suggested control strategy. Numerical simulations confirmed the effectiveness of the designed controller in suppressing the chaotic oscillation and achieving the desired objectives in different situations.

\section{Appendix}

\section{A. System Parameters}

$$
\begin{aligned}
f_{0} & =5, \\
H & =5, \\
V_{0} & =1, \\
P_{m} & =1.3, \\
x_{d}^{\prime} & =0.4, \\
x & =0.5, \\
T_{d 0}^{\prime} & =10, \\
x_{d} & =1, \\
K_{a} & =190, \\
V_{\text {ref }} & =1.05, \\
E_{\mathrm{fdr}_{\min }} & =0, \\
E_{\mathrm{fdr}_{\text {max }}} & =5, \\
E_{\mathrm{fd} 0} & =2 .
\end{aligned}
$$

\section{Data Availability}

All the numerical simulation parameters are mentioned in the respective text part, and there are no additional data requirements for the simulation results.

\section{Conflicts of Interest}

The authors declare that they have no conflicts of interest.

\section{Acknowledgments}

This study was partially funded by Center for Nonlinear Systems, Chennai Institute of Technology, India (funding no. CIT/CNS/2021/RD/022).

\section{References}

[1] F. Min, C. Yuan, G. Huang, J. Zhu, and Y. Dou, "Dynamics analysis for 3-node power system with power disturbances," in Proceedings of the 2019 22nd International Conference on Electrical Machines and Systems (ICEMS), pp. 1-6, IEEE, Harbin, China, August 2019.

[2] Y.-N. Yu, Electric Power System Dynamics, p. 1983, Academic Press, Inc., New York, NY, USA, 1983.

[3] B. A. Carreras, V. E. Lynch, I. Dobson, and D. E. Newman, "Critical points and transitions in an electric power transmission model for cascading failure blackouts," Chaos: An interdisciplinary journal of nonlinear science, vol. 12, no. 4, pp. 985-994, 2002.

[4] G. Filatrella, A. H. Nielsen, and N. F. Pedersen, "Analysis of a power grid using a kuramoto-like model," The European Physical Journal B, vol. 61, no. 4, pp. 485-491, 2008. 
[5] N. Kopell and R. Washburn, "Chaotic motions in the twodegree-of-freedom swing equations," IEEE Transactions on Circuits and Systems, vol. 29, no. 11, pp. 738-746, 1982.

[6] H.-D. Chiang, R. J. T. Ian Dobson, S. T. James, and L. FekihAhmed, "On voltage collapse in electric power systems," IEEE Transactions on Power Systems, vol. 5, no. 2, pp. 601-611, 1990.

[7] H.-D. Chiang, C.-W. Liu, P. P. Varaiya, F. F. Wu, and M. G. Lauby, "Chaos in a simple power system," IEEE Transactions on Power Systems, vol. 8, no. 4, pp. 1407-1417, 1993.

[8] R. G. Kavasseri and K. R. Padiyar, "Analysis of bifurcations in a power system model with excitation limits," International journal of bifurcation and chaos, vol. 11, no. 9, pp. 2509-2516, 2001.

[9] T. Sekine, T. Tsuji, T. Oyama, F. Magoulès, and K. Uchida, "Speedup of parallel computing by parareal method in transient stability analysis of Japanese power system," in Proceedings of the 2016 IEEE Innovative Smart Grid Technologies-Asia (ISGT-Asia), pp. 1177-1182, IEEE, Melbourne, Australia, December 2016.

[10] R.-Q Wang and J.-C. Huang, "Effects of hard limits on bifurcation, chaos and stability," Acta Mathematicae Applicatae Sinica, vol. 20, no. 3, pp. 441-456, 2004.

[11] H. Zhao, Y.-J. Ma, S.-J. Liu, G. Shi-Gen, and D. Zhong, "Controlling chaos in power system based on finite-time stability theory," Chinese Physics B, vol. 20, no. 12, Article ID 120501, 2011.

[12] A. A.-H. Abdul-Basset, F. R. Tahir, A. Ouannas, T.-C. Sun, H. Jahanshahi, and A. A. Aly, "Chaos suppressing in a threebuses power system using an adaptive synergetic control method," Electronics, vol. 10, no. 13, p. 1532, 2021.

[13] M. S. Widyan, "Controlling chaos and bifurcations of smib power system experiencing ssr phenomenon using sssc," International Journal of Electrical Power \& Energy Systems, vol. 49, pp. 66-75, 2013.

[14] S. Rezaei, "Power oscillation due to ferroresonance and subsynchronous resonance," Power System Stability, 2019.

[15] H. Gholizadeh, A. Hassannia, and A. Azarfar, "Chaos detection and control in a typical power system," Chinese Physics B, vol. 22, no. 1, Article ID 010503, 2013.

[16] I. Podlubny, "An introduction to fractional derivatives, fractional differential equations, to methods of their solution and some of their applications," Mathematics in Science and Engineering, vol. 198, pp. 223-242, 1999.

[17] B. Dumitru, K. Diethelm, E. Scalas, and J. J. Trujillo, "Fractional calculus: models and numerical methods," World Scientific, vol. 3, 2012.

[18] S. A. David, J. L. Linares, and E. M. J. A. Pallone, "Fractional order calculus: historical apologia, basic concepts and some applications," Revista Brasileira de Ensino de Física, vol. 33, no. 4, p. 4302, 2011.

[19] K. Rajagopal, A. Karthikeyan, D. Prakash, R. Weldegiorgis, and G. Tadesse, "Bifurcation, chaos and its control in a fractional order power system model with uncertainties," Asian Journal of Control, vol. 21, no. 1, pp. 184-193, 2019.

[20] S. Kumar Panda, P. K. Dewangan, and D. K. Lal, "Elimination of chaos in both integer and fractional order single-machineinfinite-bus power system using lyapunov based controller," in Proceedings of the 2019 IEEE International Conference on System, Computation, Automation and Networking (ICSCAN), pp. 1-6, IEEE, Pondicherry, India, March 2019.

[21] R. Karnatak, "Linear augmentation for stabilizing stationary solutions: potential pitfalls and their application," PLoS One, vol. 10, no. 11, Article ID e0142238, 2015.
[22] P. R. Sharma, A. Sharma, M. D. Shrimali, and A. Prasad, "Targeting fixed-point solutions in nonlinear oscillators through linear augmentation," Physical review. E, Statistical, nonlinear, and soft matter physics, vol. 83, no. 6, Article ID 067201, 2011.

[23] P. R. Sharma, M. D. Shrimali, A. Prasad, and U. Feudel, "Controlling bistability by linear augmentation," Physics Letters A, vol. 377, no. 37, pp. 2329-2332, 2013.

[24] P. R. Sharma, A. Singh, A. Prasad, and M. Dev Shrimali, "Controlling dynamical behavior of drive-response system through linear augmentation," The European Physical Journal Special Topics, vol. 2238, p. 1531, 1539.

[25] P. R. Sharma, M. Dev Shrimali, A. Prasad, V. K. Nikolay, and G. A. Leonov, "Controlling dynamics of hidden attractors," International Journal of Bifurcation and Chaos, vol. 25, no. 4, Article ID 1550061, 2015.

[26] I. Podlubny, Fractional differential equations, vol. 198 of Mathematics in Science and Engineering, Academic Press, Cambridge, MA, USA, 1999.

[27] I. Petráš, "Comments on "coexistence of hidden chaotic attractors in a novel no-equilibrium system"(nonlinear dyn)," Nonlinear Dynamics, vol. 90, no. 1, pp. 749-754, 2017.

[28] M. Saleh Tavazoei and M. Haeri, "A necessary condition for double scroll attractor existence in fractional-order systems," Physics Letters A, vol. 367, no. 1-2, pp. 102-113, 2007.

[29] B. M. Vinagre, Y. Q. Chen, and I. Petráš, "Two direct tustin discretization methods for fractional-order differentiator/ integrator," Journal of the Franklin Institute, vol. 340, no. 5, pp. 349-362, 2003.

[30] I. Petráš, Fractional-order Nonlinear Systems: Modeling, Analysis and Simulation, Springer Science \& Business Media, Berlin, Germany, 2011.

[31] V. Venkatasubramanian and W. Ji, "Coexistence of four different attractors in a fundamental power system model," IEEE Transactions on Circuits and Systems I: Fundamental Theory and Applications, vol. 46, no. 3, pp. 405-409, 1999.

[32] M. Saleh Tavazoei and M. Haeri, "Chaotic attractors in incommensurate fractional order systems," Physica D: Nonlinear Phenomena, vol. 237, no. 20, pp. 2628-2637, 2008.

[33] C. Pezeshki, S. Elgar, and R. C. Krishna, "Bispectral analysis of possessing chaotic motion," Journal of Sound and Vibration, vol. 137, no. 3, pp. 357-368, 1990.

[34] P. R. Sharma, M. D. Shrimali, A. Prasad, N. V. Kuznetsov, and G. A. Leonov, "Control of multistability in hidden attractors," The European Physical Journal Special Topics, vol. 224, no. 8, pp. 1485-1491, 2015.

[35] T. F. Fozin, G. D. Leutcho, A. T. Kouanou et al., "Multistability control of hysteresis and parallel bifurcation branches through a linear augmentation scheme," Zeitschrift für Naturforschung A, vol. 75, no. 1, pp. 11-21, 2019.

[36] N. Aguila-Camacho, M. A. Duarte-Mermoud, and J. A. Gallegos, "Lyapunov functions for fractional order systems," Communications in Nonlinear Science and Numerical Simulation, vol. 19, no. 9, pp. 2951-2957, 2014.

[37] J. Ni, L. Liu, C. Liu, and X. Hu, "Fractional order fixed-time nonsingular terminal sliding mode synchronization and control of fractional order chaotic systems," Nonlinear Dynamics, vol. 89, no. 3, pp. 2065-2083, 2017.

[38] C. Prakash, A. Banerjee and P. P. Singh, Analysis and control of chaotic oscillation in fosmib power system using aismc technique," in Proceedings of the 2019 IEEE Students Conference on Engineering and Systems (SCES), pp. 1-6, IEEE, Allahabad, India, May 2019. 
[39] T. F. Fozin, R. Kengne, J. Kengne, K. Srinivasan, M. Souffo Tagueu, and F. B. Pelap, "Control of multistability in a selfexcited memristive hyperchaotic oscillator," International Journal of Bifurcation and Chaos, vol. 29, no. 9, Article ID 1950119, 2019. 